\title{
Boolean Non-null Data Type
}

National Cancer Institute

\section{Source}

National Cancer Institute. Boolean Non-null Data Type. NCI Thesaurus. Code C95630.

A data type comprised of values that adhere to two-value logic such as True and False

but does not include a Null Flavor. 